\title{
MIXTURE OF COMPETITIVE LINEAR MODELS FOR PHASED-ARRAY MAGNETIC RESONANCE IMAGING*
}

\author{
Deniz Erdogmus ${ }^{1}$, Rui Yan ${ }^{1}$, Erik G. Larsson ${ }^{2}$, Jose C. Principe ${ }^{1}$, Jeffrey R. Fitzsimmons ${ }^{3}$ \\ ${ }^{1}$ Dept. of ECE, University of Florida, Gainesville, FL 32611, USA \\ ${ }^{2}$ Dept. of ECE, The George Washington University, Washington, DC 20052, USA \\ ${ }^{3}$ Dept. of Radiology, University of Florida, Gainesville, FL 32610, USA
}

\begin{abstract}
Phased-array magnetic resonance imaging is an important contemporary research field in terms of the expected clinical gains in medical imaging technology. Recent research focused on heuristic coil image recombination methods as well as statistical signal processing approaches. In this paper, we investigate the performance of an adaptive signal processing approach, namely mixture of competitively trained models. The proposed method has the ability to train on a set of images and generalize its performance to previously unseen images. Performance evaluations on real data validate the effectiveness of this method.
\end{abstract}

\section{INTRODUCTION}

Magnetic resonance image (MRI) image reconstruction with phased-array coils is being widely studied. In this imaging technique, the challenge is to counter the problems emerging from the unknown and spatially varying sensitivities of each coil. The Sumof-Squares(SoS) method, which introduces bias even in noise-free case, can be interpreted to estimate the coil sensitivities and implement optimal linear combination based on these estimates. It can be shown that SoS asymptotically approaches reconstruction optimality as all measurement (coil) SNR levels increase [1]. However, the high measurement SNR condition is not always satisfied in practice, especially in phased-arrays, where the coils measure only a portion of the image. This creates the problem of considering pure noise pixels equally weighted to pixels with actual signal. A number of somewhat more sophisticated techniques for image construction with phased-array coils have appeared during the last decade. For example, as an alternative to SoS reconstruction, Debbins et al. suggested adding the images coherently after their relative phase is properly adjusted [2]. Bydder et al. proposed a method that attempts to estimate the coil sensitivities from the image [3]; although the resulting image has somewhat less variance than the SoS reconstruction it still suffers from the same problems with bias. Kellman and McVeigh proposed a method that can use the degrees of freedom inherent to the phased array for ghost artifact cancellation [4]. A somewhat more sophisticated technique has been presented by Walsh et al. who used adaptive filters to improve the SNR in the image [5]. Finally, a Bayesian method using iterative maximum likelihood with prior information in coil sensitivities is presented recently by the authors [6]. All these enahncement to SoS, without exception, relied on building algorithms

${ }^{*}$ THIS WORK WAS PARTIALLY SUPPORTED BY THE NSF GRANT ECS-0300340. based on assumptions about the signal model. These approaches were either heuristic or statistical in nature. An adaptive signal processing framework has not yet been studied for phased array MRI. In this paper, we propose a first step to tackling the image reconstruction problem in multiple-coil MRI scenarios. The expected gains from this approach include the following: there is no need to discover signal models that describe the measurements well (a must in statistical signal processing approaches). Moreover, adaptive systems are more flexible and robust to inconsistencies and nonstationarities in the data as they can be updated online while in use, using a meaningful adaptation paradigm adaptive systems are as well able to approximate optimal statistical signal processing approaches (to the limits set by the topology) while requiring less design effort and computational complexity.

Specifically, this paper deals with the reconstruction of MRI images from multiple coils by a mixture of competitively trained models. As a first step, the current approach considers linear modalities in both the model and mixture stages. The training is performed using competitive LMS to minimize the MSE between the model outputs and the desired output in the training set. The performance and the generalization ability of the trained linear mixture of linear models is verified on experiments using human neck images from a 4-coil phased-array.

\section{THE LINEAR MIXTURE OF LINEAR MODELS FOR PHASED-ARRAY MRI}

Consider a phased-array MRI system with $M \times N$ pixels where each pixel contains $n_{c}$ values (corresponding to the measurements of $n_{c}$ coils for the pixel). For pixel $n, s(n)=$ $\left[s_{1}(n), \cdots, s_{n_{c}}(n)\right]^{T}$ is the vector of coil measurements for pixel $n$, where $n=1, \cdots, M N$. A commonly used standard image reconstruction method in phased-array MRI is Sum-ofSquares (SoS). In this method, the object density (or the image intensity) is estimated according to the following equation, which also takes the noise correlation across coils into account.

$$
\hat{\rho}(n)=\boldsymbol{s}(n)^{T} \boldsymbol{Q}^{-1} \boldsymbol{s}(n)
$$

Since, in general, the noise covariance matrix is not known a priori, a small region of pixels consisting purely of noise must be used to estimate it empirically. This often requires a manual selection of the noise pixels, which assmes the noise model unity globally. It is a process that is vulnerable to errors if the noise demonstrates local properties. Alternatively, in SoS reconstruction, the noise covariance matrix can be ignored and the image estimate can be reconstructed using the simpler form $\hat{\rho}(n)=\boldsymbol{s}(n)^{T} \boldsymbol{s}(n)$. According 


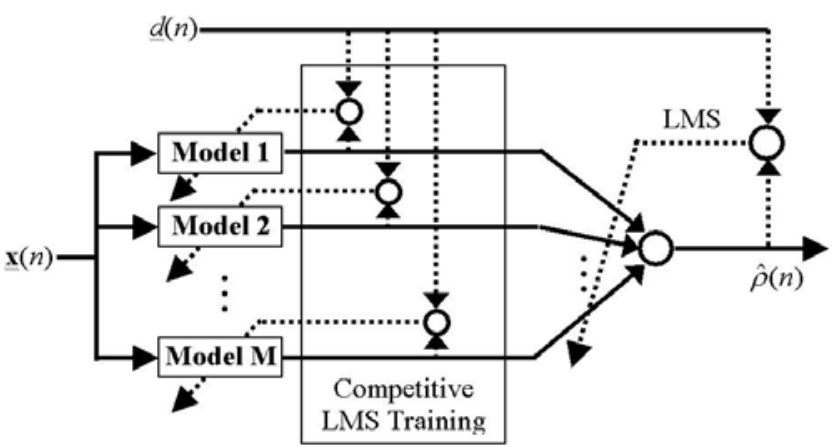

Fig. 1. Block diagram of the multiple model mixture and learning scheme.

to Roemer et al. [7], when using high SNR coil measurements, ignoring the noise covariance generates at most an additional $10 \%$ error (assuming $43 \%$ noise covariance between channels). In low SNR measurement situations, however, this might not be the case and the latter SoS reconstruction will suffer greatly from the noise correlations.

In order to circumvent these difficulties associated with SoS, and perhaps to improve the quality of the reconstructed image in terms of SNR, an adaptive mixture model approach can be employed. In this paper, we are particularly interested in a mixture of linear models. Training methodologies for mixture of models have been reviewed and investigated for image and time series segmentation previously [8]. In the case of MRI, it is possible to obtain a sequence of training (calibration) images of a certain object. The average of the reconstructed images using these measurements, which expectedly possesses much higher SNR, can be used as the desired output in the competitive framework.

Following the methodologies outlined in [8], the training of these models is performed using a set of training measurements consisting of $K$ measurements (images of the same object) taken by the phased-array system. Each of these $K$ training measurements consists of an input vector constructed by the individual coil measurements as well as the whitened SoS reconstruction (using the coil measurements) for each pixel. The desired output $d(n)$ is the same for every training sample and it is the average of whitened SoS reconstructions. The mixture model and the general training scheme are depicted in Fig. 1.

The mixture of linear models consist of multiple local experts whose outputs are $y_{i}(n)=\boldsymbol{w}_{i}^{T} \boldsymbol{x}(n)$ for $i=1, \cdots, M$ where $\mathrm{M}$ is the number of linear models and $\boldsymbol{x}(n)=$ $\left[s_{1}(n), \cdots, s_{n_{c}}(n), \hat{\rho}_{S o S}(n)\right]^{T}$, where $\rho_{S o S}(n)$ is calculated using (1) with the noise covariance estimated from a purely noise region of the coil images. These linear models are trained competitively using LMS [9] (in a winner-take-all fashion), where the criterion of the competition is the prediction MSE of the desired outputs for a $l \times l$ region centered at the $n^{t h}$ pixel.

$$
\begin{gathered}
\boldsymbol{w}_{k}(n+1)=\boldsymbol{w}_{k}(n)+\boldsymbol{\mu}_{1} \boldsymbol{x}(n) e_{1}(n) \\
e_{1}(n)=d(n)-\boldsymbol{w}_{k}(n)^{T} \boldsymbol{x}(n)
\end{gathered}
$$

where $k=\arg \min _{k} \sum_{j=1}^{l^{2}}\left(d(j)-y_{k}(j)\right)^{2}$ is the winning model index $k, l^{2}$ is the number of pixels in the local region and $\mu_{1}$ is the step size.

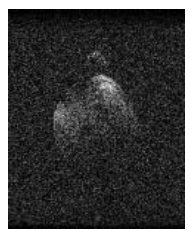

(a) Coil 1

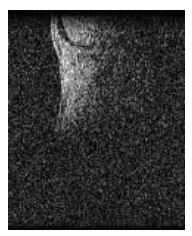

(e) Coil 1

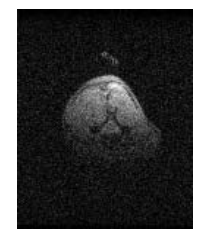

(b) Coil 2

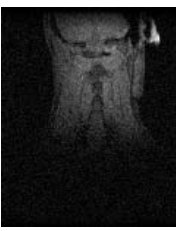

(f) Coil 2

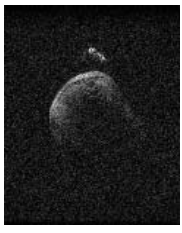

(c) Coil 3

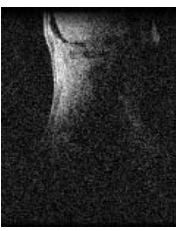

(g) Coil 3

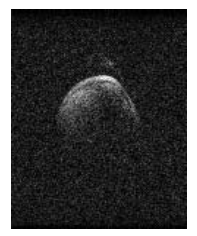

(d) Coil 4

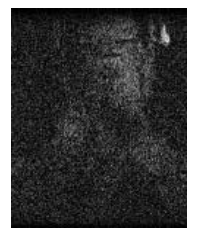

(h) Coil 4
Fig. 2. Transverse (upper row) and coronal (lower row) crossections of a human neck as measured by the four coils.

The multiple model outputs are then combined to produce an estimate of the image intensity at the $n^{\text {th }}$ pixel using

$$
\hat{\rho}(n)=\boldsymbol{g}(n)^{T} \boldsymbol{y}(n)
$$

where $\boldsymbol{y}(n)=\boldsymbol{W}^{T} \boldsymbol{x}(n)$ is the vector of outputs from the multiple models, and the mixing weights are also linear combinations of the input, i.e., $\boldsymbol{g}(n)=\boldsymbol{V} \boldsymbol{x}(n)$. Once the multiple linear models are trained with competitive LMS, the mixing matrix parameters $\boldsymbol{V}$ can be trained with LMS using the outputs of the competitive models as the input and the same desired output.

$$
\begin{aligned}
\boldsymbol{V}(n+1) & =\boldsymbol{V}(n)+\mu_{2} \boldsymbol{y}(n) \boldsymbol{x}(n)^{T} e_{2}(n) \\
e_{2}(n) & =d(n)-\boldsymbol{x}(n)^{T} \boldsymbol{V}^{T} \boldsymbol{y}(n)
\end{aligned}
$$

where $\mu_{2}$ is the step size.

Since, in this paper, we assumed that both linear models and mixture weights are linear functions of the inputs, in the test phase where a previously unseen set of measurements are taken to image an object, the output of the proposed mixture of linear experts can be written as $\hat{\rho}(n)=\boldsymbol{x}(n)^{T} \boldsymbol{V}^{T} \boldsymbol{W}^{T} \boldsymbol{x}(n)=\boldsymbol{x}(n)^{T} \boldsymbol{G} \boldsymbol{x}(n)$. We note that this final form to reconstruct images in the test phase is similar to the whitened SoS reconstruction given in (1), except the weighting matrix is trained using the MSE criterion and the multiple model concept over a training (calibration) set. The adaptive approach has the advantage that if the noise is not spatially stationary, the local models will specialize to different modalities of the noise and the adaptive mixture model will still be able to produce high SNR reconstructions reliably.

\section{NUMERICAL RESULTS}

In this section, we demonstrate the performace of the proposed mixture model approach in phased-array MRI reconstruction using transverse (45 measurements) and coronal (9 measurements) human neck images collected by a 4-coil MRI system. Sample 
measurements from the four coils for both crossections are shown in Fig. 2. All transverse crossection measurements are used for training and one of the coronal crossection measurements (the one that is shown in Fig. 2) is used for testing the resulting network.

The coil measurements of the test image (coronal crossection) are combined using SoS (without and with whitening) as well as the proposed mixture model network. The resulting reconstructed images and their local SNR levels are shown in Fig. 3\&4. Especially the SNR levels in regions where signal exists are important. Larger SNR at these regions indicate better performance, whereas the SNR levels in the purely noise regions are not significant due to the computation method for this quality measure. Since a reference (a ground truth) is not available in MRI, typically the image quality is measured by this measure called SNR, which in fact does not conform to the traditional definition of SNR in signal processing. The procedure for computing the $S N R$ is as follows:

1. Find a reference region in the reconstructed image where there is no signal (i.e., a pure noise region).

2. Compute the variance of the noise in this reference region.

3 . For all other regions, compute the signal power (which includes both the actual signal and the remaining noise in that region).

4. Calculate SNR in a region as the ratio of the power of the signal in that region to the variance of the noise in the reference region. Convert SNR to $\mathrm{dB}$.

From Fig. 3\&4, focusing only on the SNR levels in the signal regions, we observe that the mixture of models approach improved the performance upto $2 \mathrm{~dB}$ and $12 \mathrm{~dB}$ over that of the SoS with whitening and SoS without whitening methods, respectively. The gray region at the upper-left corner is used as the noise reference in computing the SNR levels.

\section{CONCLUDING REMARKS}

The sum-of-squares algorithm is a very simple and practical approach in phased-array MRI that yields acceptable image reconstructions from high SNR measurements. The quality of the SoS reconstruction, however, is vulnerable to the measurement noise levels in each coil as it is common to have phased-arrays such that the coil arrangement leads to measurements that specialize to certain regions of the images. In such cases, for a given pixel, some coils will measure only noise, yet SoS will consider them as valid measurements in the combination.

In this paper, we proposed a mixture of multiple models approach for phased-array MRI, which can circumvent this and other possible problems that might influence the SoS reconstruction quality (such as noise non-stationarity). This method relies on the local approximation capabilities of the adaptive models, thus its performance can be improved by modifying the modeling units properly. As an initial step, here we investigated the performance of linear local models combined with a linear weighting scheme and showed $2 \mathrm{~dB}$ SNR improvement upon the best performance that can be achieved by the SoS method.

It is concievable that further improvement of the performance is possible by considering nonlinear models combined with nonlinear mixing schemes. In addition, the competitive training strategy that is followed in training the multiple models help to create image reconstruction methods that are more robust to spatial nonstationarities in measurements. Furthermore, under the described framework, a continuously learning image reconstruction scheme can be obtained since every testing measurement can as well be utilized as a training sample to update the weights of the mixture and the multiple models. This can also help account for temporal nonstationarities in the parameters of the MRI equipment and the signal and noise characteristics.

The phased-array MRI research has mostly focused on signal processing techniques that stem from heuristics of more traditional statistical techniques. There is very little work that incorporates the adaptive signal processing techniques to the solution of this important and practical problem. Certainly, there is much to be discovered in terms of benefits of applying adaptive signal processing techniques to phased-array MRI.

Acknowledgments: The authors would like to thank Jim Caserta, Dave Peterson and Barbara Beck at the Mcknight Brain Institute of the University of Florida for the data collection and valuable discussions.

\section{REFERENCES}

[1] E. G. Larsson, R. Yan, D. Erdogmus, J.C. Principe, and J.R. Fitzsimmons, "Snr-optimality of sum-of-squares reconstruction for phased-array magnetic resonance imaging," to appear in Journal of Magnetic Resonance, 2003.

[2] J P Debbins, J P Felmlee, and S J Riederer, "Phase alignment of multiple surface coil data for reduced bandwidth and reconstruction requirements," Magnetic Resonance in Medicine, vol. 38, pp. 1003-1011, 1997.

[3] M Bydder, D J Larkman, and J V Hajnal, "Combination of signals from array coils using image-based estimation of coil sensitivity profiles," Magnetic Resonance in Medicine, vol. 47, pp. 539-548, 2002.

[4] P Kellman and E R McVeigh, "Ghost artifact cancellation using phased array processing," Magnetic Resonance in Medicine, vol. 46, pp. 335-343, 2001.

[5] D O Walsh, A F Gmitro, and M W Marcellin, "Adaptive reconstruction of phased array MR imagery," Magnetic Resonance in Medicine, vol. 43, pp. 682-690, 2000.

[6] R. Yan, D. Erdogmus, E.G. Larsson, J.C. Principe, and J.R. Fitzsimmons, "Image combination for high-field phased-array mri," in Proceedings of ICASSP03, Apr 2003.

[7] P B Roemer, W A Edelstein, C E Hayes, S P Souza, and O M Mueller, "The NMR phased array," Magnetic Resonance in Medicine, vol. 16, pp. 192-225, 1990.

[8] G. L. Fancourt, Gated Competitive Systems for Unsupervised Segmentation and Modeling of Piecewise Stationary Signals, Ph.D. thesis, University of Florida, Gainesville, FL, USA, 1998, To be published.

[9] S. Haykin, Adaptive Filter Theory, Pearson Education, Inc., Delhi, India, 2002. 

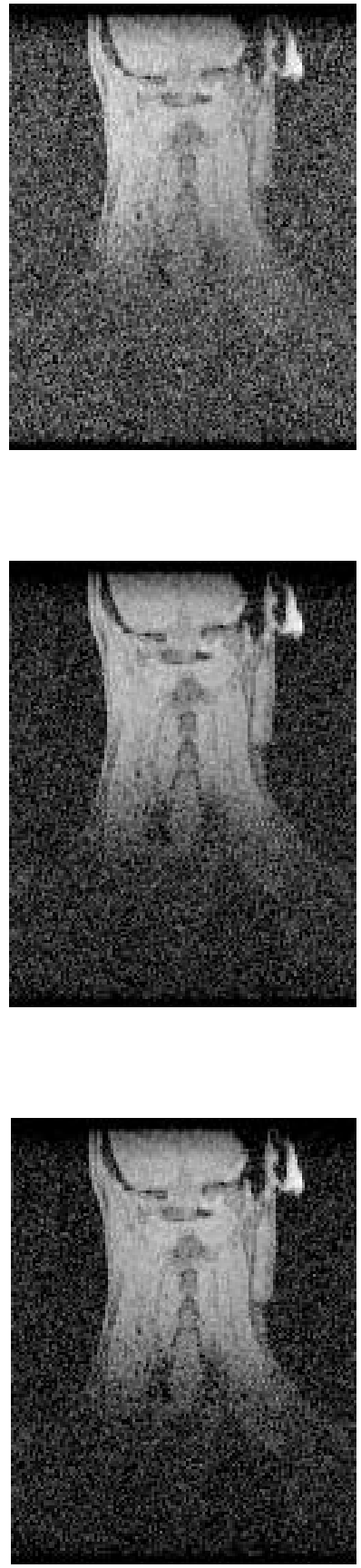

Fig. 3. Reconstructed test images for a coronal crossection from a human neck, (a) SoS without whitening (b) SoS with whitening (c) mixture of models.
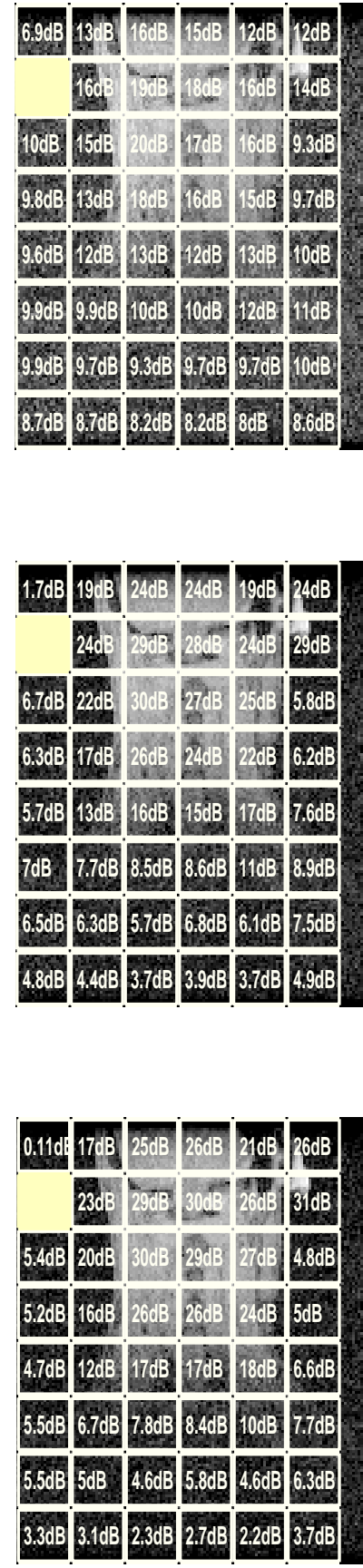

Fig. 4. SNR performances of the reconstructed test images for a coronal crossection from a human neck, (a) SoS without whitening (middle row) SoS with whitening (c) mixture of models. 